\title{
A rare cause of vomiting in an adolescent: gastric Burkitt's lymphoma
}

\author{
Derya Altay ${ }^{1}$, Alper Özcan², Ekrem Ünal², Kemal Deniz³, Keramettin Uğur Özkan ${ }^{4}$, \\ Türkan Patıroğlu ${ }^{2}$, Duran Arslan ${ }^{1}$ \\ Departments of ${ }^{1}$ Pediatric Gastroenterology, Hepatology and Nutrition, ${ }^{2}$ Pediatric Hematology and Oncology, ${ }^{3}$ Pathology \\ and ${ }^{4}$ Pediatric Surgery, Erciyes University Faculty of Medicine, Kayseri, Turkey. E-mail: dr.deryaaltay@gmail.com \\ Received: 19th October 2018, Revised: 12th November 2018, 7th December 2018, Accepted: 7th December \\ 2018
}

SUMMARY: Altay D, Özcan A, Ünal E, Deniz K, Özkan KU, Patıroğlu T, Arslan D. A rare cause of vomiting in an adolescent: gastric Burkitt's lymphoma. Turk J Pediatr 2019; 61: 431-435.

Burkitt's lymphoma, which is the most commonly diagnosed pediatric gastrointestinal tumor, usually affects the intestinal tract. However, the diagnosis of gastric Burkitt's lymphoma in childhood is extremely rare. Here, we have reported the case of an adolescent male patient with vomiting and weight loss symptoms who was diagnosed with Burkitt's lymphoma of the pyloric antrum. The patient responded very well to chemotherapy and he did not develop tumor lysis syndrome. Based on this case report, gastric Burkitt's lymphoma might be included in the differential diagnoses of pediatric patients who experience vomiting that results in significant weight loss.

Key words: adolescent, Burkitt's lymphoma, gastric.

Burkitt's lymphoma is a B-cell non-Hodgkin's lymphoma; therefore, it is a fast-growing and highly aggressive tumor. There are three clinical variants of Burkitt's lymphoma: endemic, sporadic, and immunodeficiency-related. Sporadic Burkitt's lymphoma accounts for 30$50 \%$ of all childhood lymphomas, and it occurs in children between the ages of 3 and 12 years old. The symptoms of Burkitt's lymphoma include abdominal pain, distension, nausea, vomiting, and gastrointestinal bleeding. ${ }^{1-3}$ Due to its rapid growth, tumor lysis syndrome may occur in the process of diagnosis and treatment. As a result of the rapid growth of Burkitt's lymphoma, it has a tendency toward exhibiting obstructive effects in the lumens of the affected organs, such as the stomach and bowels. However, it is sensitive to and responds well to chemotherapy. ${ }^{4}$

Burkitt's lymphoma frequently involves the terminal ileum region, where the lymphatic tissue in the gastrointestinal tract is the most intense; however, colonic and gastric involvement are extremely rare. ${ }^{5}$ Asymmetrical colonic wall thickening, solid hypoechoic mass nodules, ascites or lymphadenopathy can all be diagnosed using ultrasonography, but computed tomography scans reveal better views of the findings. ${ }^{5,6}$ Here, we have presented the case of an adolescent patient with vomiting symptoms who was ultimately diagnosed with gastric Burkitt's lymphoma.

\section{Case Report}

A 13-year-old male presented as an outpatient with complaints of vomiting after every meal, resulting in a loss of $10 \mathrm{~kg}$ over the course of three months. The patient reported that the emesis products were brown in color, and he reported black-colored feces that started when he began experiencing the symptoms. He complained of localized stomach pain, but he did not report a bitter taste in his mouth. During the physical examination, the patient's weight was $42 \mathrm{~kg}$ (z score: - 0.19), his height was $164 \mathrm{~cm}$ (z score: 1.37), and his abdomen was normally shaped. The laboratory results were as follows, hemoglobin: $9 \mathrm{~g} / \mathrm{dL}$, mean 
corpuscular volume: $71.8 \mathrm{fL}$, iron: $17 \mathrm{mcg} /$ $\mathrm{dL}$ (normal range: $59-158 \mathrm{mcg} / \mathrm{dL}$ ), ferritin: $4.2 \mathrm{ng} / \mathrm{mL}$ (normal range: 14-124 ng/mL), vitamin B12: $147 \mathrm{pg} / \mathrm{mL}$ (normal range: 183$1090 \mathrm{pg} / \mathrm{mL}$ ), and lactate dehydrogenase: 348 IU/L (normal range: 135-225 IU/L). The other biochemical parameters were within normal limits.

Upper gastrointestinal endoscopic evaluation revealed a mucosal lesion with protrusive edema and fragile, hyperemic ulcers at the pyloric antrum, from the duodenal bulb through the stomach. Although the bulb exhibited widespread ulceration, the second part of the duodenum was normal (Fig. 1). The histopathology of the lesion revealed hyperplastic changes. The patient was treated with proton pump inhibitors, but his vomiting resumed. One week later, a second upper gastrointestinal endoscopy was performed. The results showed a polypoid lesion growth, that was blocking passage from the pylorus; therefore, the second part of the duodenum could not be viewed. The biopsies from the second endoscopic evaluation of the lesion also exhibited hyperplastic changes. Subsequently, an abdominal ultrasonography scan showed a $2.5-\mathrm{cm}$ thickening of the stomach wall, resulting in luminal narrowing of the pyloric opening, continuing on to the inferior part of the stomach, and a solid mass of $6 \times 4.5 \mathrm{~cm}$ was observed. In the diffusion-weighted images, the contrast-enhanced magnetic resonance imaging showed a mass lesion with diffusion limitations in the gastric outlet (Fig. 2). A deep tissue biopsy was performed by the Pediatric Surgery Department, in addition to a percutaneous endoscopic jejunostomy. The histological examination of the gastric biopsy samples revealed a medium-sized, atypical lymphoid cell infiltration, with a coarse chromatin pattern, and cells with narrow cytoplasm. The visual scan of the slides revealed a sporadic starry sky pattern. The immunohistochemical examination revealed CD20 positive, CD10 positive, Bcl6 positive reactions in the neoplastic cells. The Ki67 proliferation index was $100 \%$. The fluorescent in situ hybridization results were positive, with translocation in $50 \%$ of the cells when using the Cmyc break-apart probe (Fig. 3).

This patient was transferred to the Children's Oncology Department for chemotherapy. A bone marrow aspiration was conducted, but it did not reveal any blastic cells. However, a positron emission tomography scan revealed diffuse hyperactivity and gastric mucosa thickening. The patient was treated according to the Berlin-Frankfurt-Munster Group NHLBFM-90 protocol. ${ }^{7}$ During the NHL-BFM-90 treatment, the patient began oral feeding (he was being fed previously via the jejunostomy). Subsequently, after the patient was consuming an adequate amount of food through his oral intake, the jejunostomy was removed due to the increased risk of infection at the jejunostomy site. The biochemical values determining tumor lysis syndrome were closely monitored, and no incidence of tumor lysis syndrome developed during his chemotherapy treatments. This

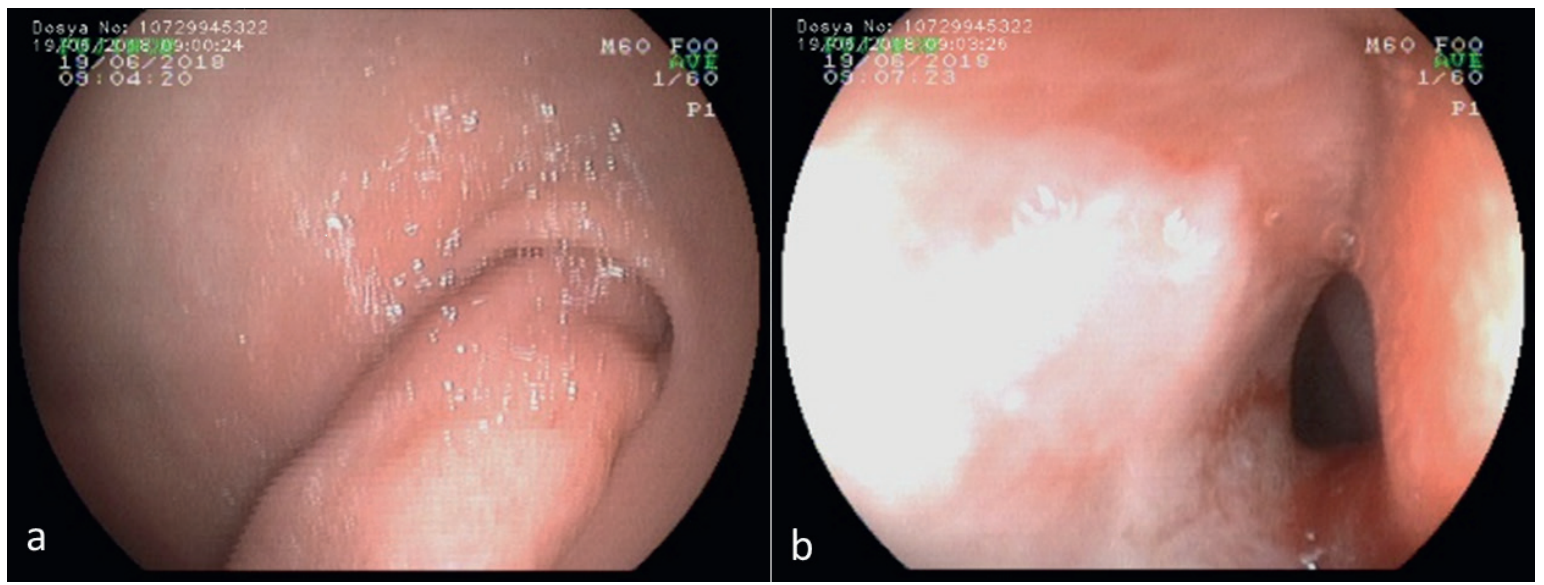

Fig. 1. a) A mucosal lesion that was prolapsed from the bulb to the pylorus and b) permitted the passage of the endoscope. 


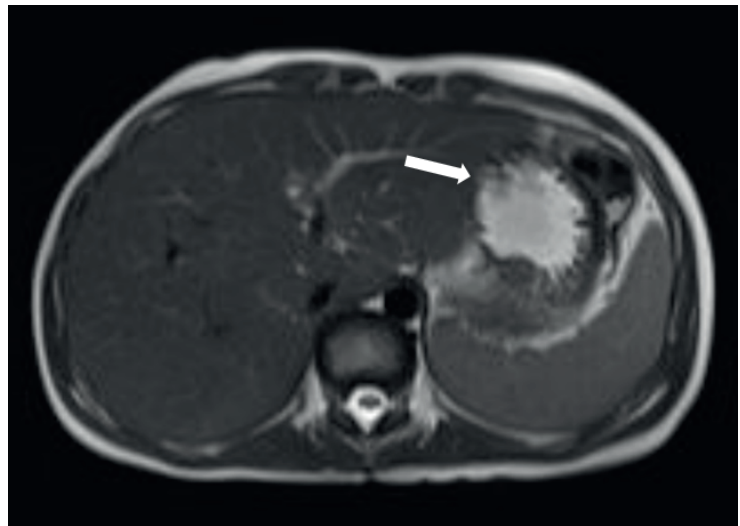

Fig. 2. The appearance of the mass on contrast MRI.

patient showed partial response after the AA and $\mathrm{BB}$ treatment courses, so the treatment was intensified with course of CC. ${ }^{7}$ After the CC course, the patient showed complete remission. This patient has already received the last planned course of treatment, and he was in complete remission, without any sequelae, at the time this manuscript was written.

Written consent was obtained from the family for the publication of this case report.

\section{Discussion}

Non-Hodgkin's lymphoma is the most commonly seen malignancy in the gastrointestinal tract of children, and $75 \%$ of these cases are diagnosed with Burkitt's lymphomas. ${ }^{8}$ Endemic Burkitt's lymphoma has been associated with Epstein-Barr virus, human immunodeficiency virus, and Plasmodium falciparum malaria infections; however, the risk factors for sporadic Burkitt's lymphoma are unknown. Mbulaiteye et al. ${ }^{9}$ suggested that an early childhood exposure to infection, male gender, and being Caucasian could be risk factors for sporadic Burkitt's lymphoma in the United States.
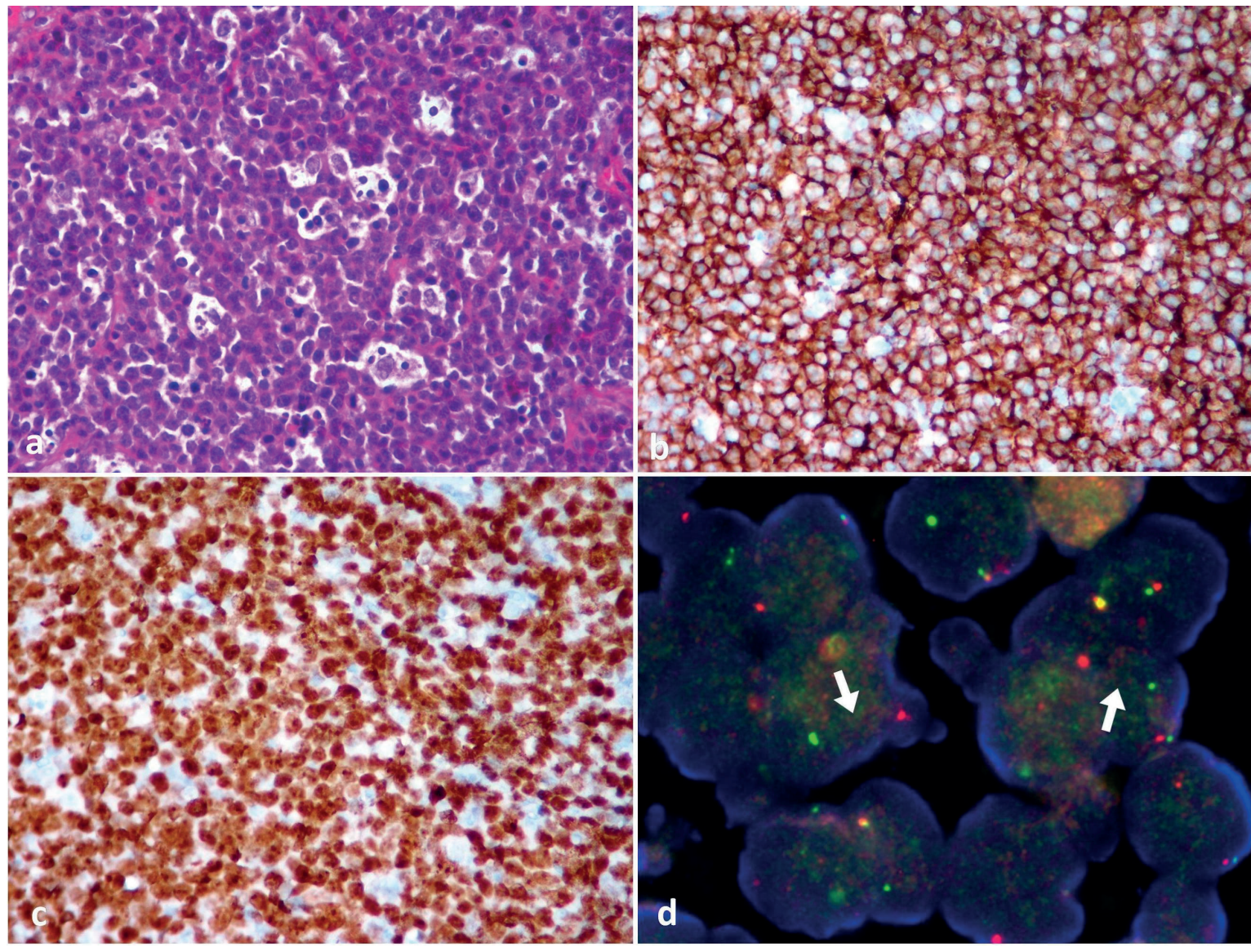

Fig. 3. a. Moderate atypical lymphoid cell infiltration in light microscope and starry sky view (hematoxylineosin, x400), b. membranous CD20 positivity in atypical lymphoid cells (x400), c. high Ki67 proliferation index (x400) and d. Myc positivity in these cells (arrows). 
In a study of 63 pediatric patients with Burkitt's lymphoma, Ertem et al. ${ }^{10}$ reported that Burkitt's lymphoma ranged from endemic to sporadic in Turkish children in terms of their epidemiological and clinical presentations, with regard to their age distribution, and due to the high incidence of abdominal involvement. Sporadic Burkitt's lymphoma often presents within the abdomen, especially in the small and large intestines in children, but gastric Burkitt's lymphoma is rarely seen in childhood. ${ }^{9,11}$ For example, Moschovi et al. ${ }^{12}$ found that only 2 (1.48\%) of 135 nonHodgkin's lymphoma cases presented as gastric Burkitt's lymphoma. They also reported that an immune response to CagA or the other Helicobacter pylori proteins may play a role in the development of high-grade lymphoma; therefore, there may be a correlation between them. In our patient's upper gastrointestinal endoscopic evaluation, the examination of the biopsy specimens taken from the antral mucosa were $\mathrm{H}$. pylori negative. During an endoscopic evaluation, lymphoma may appear nodular, similar to a reactive lymph node, or it may exhibit mucosal ulceration, hyperplasia, polyps, or infiltrative lesions. ${ }^{13}$ The endoscopic evaluation of our case showed that the mucosal ulceration was due to a polypoid lesion, and the endoscopic biopsy specimen was interpreted as exhibiting hyperplastic changes. The diagnosis of Burkitt's lymphoma was confirmed by a deep tissue biopsy. Although only one week passed between the two biopsies, the lumen was completely obstructed in the second endoscopy. This was an example of the rapid growth of Burkitt's lymphoma.

The symptoms of patients with gastric Burkitt's lymphoma include abdominal pain, nausea, vomiting, and bowel obstructions. ${ }^{12}$ The chief complaint of our patient was vomiting, and he exhibited significant weight loss as a result of the vomiting. Moreover, the color of the emesis products from our patient were sometimes brown, indicating the presence of gastrointestinal bleeding. It was assumed that the anemia seen in our patient, who was malnourished and vomiting continuously, developed secondarily to his iron and vitamin deficiencies. Fortunately, this patient exhibited no bone marrow involvement.
Burkitt's lymphoma responds very well to chemotherapy; therefore, it usually has a good prognosis. In our case, a jejunostomy was placed due to the stomach obstruction. During early stages of chemotherapy, the patient began to take in food orally, reaching his daily caloric needs in a very short time. Patients with Burkitt's lymphoma have an increased risk of tumor lysis syndrome before and during the chemotherapy process. ${ }^{15}$ However, our patient did not develop tumor lysis syndrome.

Gastric Burkitt's lymphoma occurs rarely in childhood; however, it might be considered in the differential diagnosis of patients who present with vomiting that results in weight loss.

\section{REFERENCES}

1. Molyneux EM, Rochford R, Griffin B, et al. Burkitt's's lymphoma. Lancet 2012; 379: 1234-1244.

2. Pannone G, Zamparese R, Pace $M$, et al. The role of EBV in the pathogenesis of Burkitt's lymphoma: an Italian hospital based survey. Infect Agent Cancer 2014; 9: 34.

3. Huang $\mathrm{H}$, Liu ZL, Zeng $\mathrm{H}$, et al. Clinicopathological study of sporadic Burkitt's lymphoma in children. Chin Med J (Engl) 2015; 128: 510-514.

4. Ghoroubi J, Mirshemirani A, Kouranloo J, Nazari S Abdominal Burkitt's's lymphoma in children. Iran J Pediatr Surg 2015; 1: 28-33.

5. Derinkuyu BE, Boyunağa Ö, Öztunalı Ç, et al. Imaging features of Burkitt's lymphoma in pediatric patients. Diagn Interv Radiol 2016; 22: 95-100.

6. Biko DM, Anupindi SA, Hernandez A, Kersun L, Bellah R. Childhood Burkitt's lymphoma: abdominal and pelvic imaging findings. AJR Am J Roentgenol 2009; 192: 1304-1315.

7. Reiter A, Schrappe M, Tiemann M, et al. Improved treatment results in childhood B-cell neoplasms with tailored intensification of therapy: a report of the Berlin-Frankfurt-Munster Group Trial NHL-BFM-90. Blood 1999; 94: 3294-3306.

8. Kapitancuke M, Vasciunaite A, Augustiniene R, Sakalinskiene J, Kleinotiene G. Primary gastric Burkitt's's lymphoma-induced anaemia: a case report and literature review. Acta Med Litu 2018; 25: 23-30.

9. Mbulaiteye SM, Biggar RJ, Bhatia K, Linet MS, Devesa SS. Sporadic childhood Burkitt lymphoma incidence in the United States during 1992-2005. Pediatr Blood Cancer 2009; 53: 366-370.

10. Ertem U, Duru F, Pamir A, et al. Burkitt's lymphoma in 63 Turkish children diagnosed over a 10 year period. Pediatr Hematol Oncol 1996; 13: 123-134. 
11. Saber MM, Zeeneldin AA, Samra MA, Farag SA. Primary gastrointestinal lymphoma in an Egyptian district: A study using a population-based cancer registry. J Egypt Natl Canc Inst 2013; 25: 95-101.

12. Moschovi M, Menegas D, Stefanaki K, Constantinidou CV, Tzortzatou-Stathopoulou F. Primary gastric Burkitt lymphoma in childhood: associated with Helicobacter pylori? Med Pediatr Oncol 2003; 41: 444-447.
13. Masoodi I. Burkitt's lymphoma of stomach: report of a case and literature review. Ann British Med Sci 2018; 4: 3-6.

14. Cairo MS, Sposto R, Perkins SL, et al. Burkitt's and Burkitt's-like lymphoma in children and adolescents: a review of the Children's Cancer Group experience. Br J Haematol 2003; 120: 660-670.

15. Senbanjo IO. Tumor lysis and acute renal failure in Burkitt's lymphoma: a review on pathophysiology and management. Indian J Nephrol 2009; 19: 83-86. 